Algebraic 83 Geometric $\mathcal{T}_{\text {opology }}$

Volume 4 (2004) 829-839

Published: 7 October 2004

ATG

\title{
Implications of the Ganea Condition
}

\author{
NORIO IWASE \\ Donald StANLEY \\ JEFFREY STROM
}

\begin{abstract}
Suppose the spaces $X$ and $X \times A$ have the same LusternikSchnirelmann category: $\operatorname{cat}(X \times A)=\operatorname{cat}(X)$. Then there is a strict inequality $\operatorname{cat}(X \times(A \rtimes B))<\operatorname{cat}(X)+\operatorname{cat}(A \rtimes B)$ for every space $B$, provided the connectivity of $A$ is large enough (depending only on $X$ ). This is applied to give a partial verification of a conjecture of Iwase on the category of products of spaces with spheres.
\end{abstract}

AMS Classification 55M30

Keywords Lusternik-Schnirelmann category, Ganea conjecture, product formula, cone length

\section{Introduction}

The product formula $\operatorname{cat}(X \times Y) \leq \operatorname{cat}(X)+\operatorname{cat}(Y)$ [1] is one of the most basic relations of Lusternik-Schnirelmann category. Taking $Y=S^{r}$, it implies that $\operatorname{cat}\left(X \times S^{r}\right) \leq \operatorname{cat}(X)+1$ for any $r>0$. In [5], Ganea asked whether the inequality can ever be strict in this special case. The study of the 'Ganea condition' $\operatorname{cat}\left(X \times S^{r}\right)=\operatorname{cat}(X)+1$ has been, and remains, a formidable challenge to all techniques for the calculation of Lusternik-Schnirelmann category. In fact, it was only recently that techniques were developed which were powerful enough to identify a space which does not satisfy the Ganea condition [8] (see also 9, 12]). It is still not well understood exactly which spaces $X$ do not satisfy the Ganea condition, although it has been conjectured that they are precisely those spaces for which $\operatorname{cat}(X)$ is not equal to the related invariant $\operatorname{Qcat}(X)$ (see [14, 17]).

Since the failure of the Ganea condition appears to be a strange property for a space to have, it is reasonable to expect that such failure would have useful and interesting implications. In this paper we explore some of the implications of the equation $\operatorname{cat}(X \times A)=\operatorname{cat}(X)$ for general spaces $A$, and for $A=S^{r}$ in particular. 
A brief look at the method of the paper [8] will help to put our results into proper perspective. The new techniques begin with the following question: if $Y=X \cup_{f} e^{t+1}$, the cone on $f: S^{t} \rightarrow X$, then how can we tell if $\operatorname{cat}(Y)>$ $\operatorname{cat}(X)$ ? It is shown (see [9, Thm. 5.2] and [12, Thm. 3.6]) that, if $t \geq \operatorname{dim}(X)$, then $\operatorname{cat}(Y)=\operatorname{cat}(X)+1$ if and only if a certain Hopf invariant $\mathcal{H}_{s}(f)$ (which is a set of homotopy classes) does not contain the trivial map $*$. It is also shown [9, Thm. 3.8] that if $* \in \Sigma^{r} \mathcal{H}_{s}(f)$, then $\operatorname{cat}\left(Y \times S^{r}\right) \leq \operatorname{cat}(X)+1$. Thus $Y$ does not satisfy Ganea's condition if $* \notin \mathcal{H}_{s}(f)$, but there is at least one $h \in \mathcal{H}_{s}(f)$ such that $\Sigma^{r} h \simeq *$.

Of course, if $\Sigma^{r} h \simeq *$, then $\Sigma^{r+1} h \simeq *$ as well, and this suggests the following conjecture (formulated in [8, Conj. 1.4]):

Conjecture If $\operatorname{cat}\left(X \times S^{r}\right)=\operatorname{cat}(X)$, then $\operatorname{cat}\left(X \times S^{r+1}\right)=\operatorname{cat}(X)$.

In this paper we prove that this conjecture is true, provided $r$ is large enough.

Theorem 1 Suppose $X$ is a $(c-1)$-connected space and let $r>\operatorname{dim}(X)-$ $c \cdot \operatorname{cat}(X)+2$. If $\operatorname{cat}\left(X \times S^{r}\right)=\operatorname{cat}(X)$, then

$$
\operatorname{cat}\left(X \times S^{t}\right)=\operatorname{cat}(X)
$$

for all $t \geq r$.

The conjecture remains open for small values of $r$.

Our main result is much more general: it shows how the equation cat $(X \times A)=$ $\operatorname{cat}(X)$ governs the Lusternik-Schnirelmann category of products of $X$ with a vast collection of other spaces.

Theorem 2 Let $X$ be a $(c-1)$-connected space and let $A$ be $(r-1)$-connected with $r>\operatorname{dim}(X)-c \cdot \operatorname{cat}(X)+2$. If $\operatorname{cat}(X \times A)=\operatorname{cat}(X)$ then

$$
\operatorname{cat}(X \times(A \rtimes B))<\operatorname{cat}(X)+\operatorname{cat}(A \rtimes B)
$$

for every space $B$.

Here $A \rtimes B=(A \times B) / B$ is the half-smash product of $A$ with $B$. When $A$ is a suspension, the half-smash product decomposes as $A \rtimes B \simeq A \vee(A \wedge B)$ (see, for example, [12, Lem. 5.9]), so we obtain the following.

Corollary Under the conditions of Theorem 2, if $A$ is a suspension, then

$$
\operatorname{cat}(X \times(A \wedge B))=\operatorname{cat}(X)
$$


for every space $B$.

Our partial verification of the conjecture is an immediate consequence of this corollary: it the special case $A=S^{r}$ and $B=S^{t-r}$.

Organization of the paper In Section 1 we recall the necessary background information on homotopy pushouts, cone length and Lusternik-Schnirelmann category. We introduce an auxiliary space and establish its important properties in Section 2. The proof of Theorem 2 is presented in Section 3.

\section{Preliminaries}

In this paper all spaces are based and have the pointed homotopy type of CW complexes; maps and homotopies are also pointed. We denote by $*$ the one point space and any nullhomotopic map. Much of our exposition uses the language of homotopy pushouts; we refer to [1] for the definitions and basic properties.

\section{$1.1 \quad$ Homotopy Pushouts}

We begin by recalling some basic facts about homotopy pushout squares. We call a sequence $A \rightarrow B \rightarrow C$ a cofiber sequence if the associated square

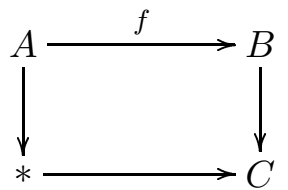

is a homotopy pushout square. The space $C$ is called the cofiber of the map $f$. One special case that we use frequently is the half-smash product $A \rtimes B$, which is the cofiber of the inclusion $B \rightarrow A \times B$.

Finally, we recall the following result on products and homotopy pushouts.

Proposition 3 Let $X$ be any space. Consider the squares
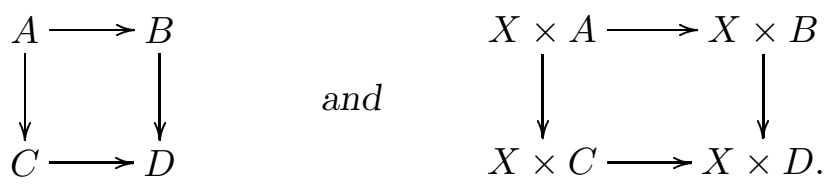

If the first square is a homotopy pushout, then so is the second.

Proof This follows from Theorem 6.2 in 15 . 


\subsection{Cone Length and Category}

A cone decomposition of a space $Y$ is a diagram of the form

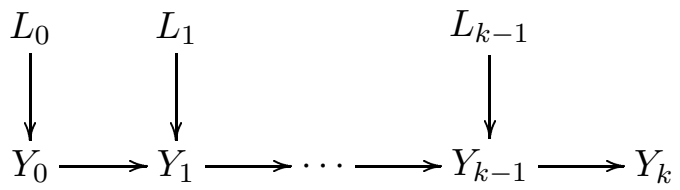

in which $Y_{0}=*$, each sequence $L_{i} \rightarrow Y_{i} \rightarrow Y_{i+1}$ is a cofiber sequence, and $Y_{k} \simeq Y$; the displayed cone decomposition has length $k$. The cone length of $Y$, denoted $\operatorname{cl}(Y)$, is defined by

$$
\operatorname{cl}(Y)= \begin{cases}0 & \text { if } Y \simeq * \\ \infty & \text { if } Y \text { has no cone decomposition, and } \\ k & \text { if the shortest cone decomposition of } Y \text { has length } k .\end{cases}
$$

The Lusternik-Schnirelmann category of $X$ may be defined in terms of the cone length of $X$ by the formula

$$
\operatorname{cat}(X)=\inf \{\operatorname{cl}(Y) \mid X \text { is a homotopy retract of } \mathrm{Y}\} .
$$

Berstein and Ganea proved this formula in [3, Prop. 1.7] with $\operatorname{cl}(Y)$ replaced by the strong category of $Y$; the formula above follows from another result of Ganea - strong category is equal to cone length [7. It follows directly from this definition that if $X$ is a homotopy retract of $Y$, then $\operatorname{cat}(X) \leq \operatorname{cat}(Y)$. The reader may refer to [10] for a survey of Lusternik-Schnirelmann category.

The category of $X$ can be defined in another way that is essential to our work. Begin by defining the $0^{\text {th }}$ Ganea fibration sequence $F_{0}(X) \longrightarrow G_{0}(X) \stackrel{p_{0}}{\longrightarrow} X$ to be the familiar path-loop fibration sequence $\Omega(X) \longrightarrow \mathcal{P}(X) \longrightarrow X$. Given the $n^{\text {th }}$ Ganea fibration sequence

$$
F_{n}(X) \longrightarrow G_{n}(X) \stackrel{p_{n}}{\longrightarrow} X,
$$

let $\bar{G}_{n+1}(X)=G_{n}(X) \cup C F_{n}(X)$ be the cofiber of $p_{n}$ and define $\bar{p}_{n+1}$ : $\bar{G}_{n+1}(X) \rightarrow X$ by sending the cone to the base point of $X$. The $(n+1)^{\text {st }}$ Ganea fibration $p_{n+1}: G_{n+1}(X) \rightarrow X$ results from converting the map $\bar{p}_{n+1}$ to a fibration. The following result is due to Ganea (cf. Svarc).

Theorem 4 For any space $X$,

(a) $\operatorname{cl}\left(G_{n}(X)\right) \leq n$,

(b) the map $p_{n}: G_{n}(X) \rightarrow X$ has a section if and only if cat $(X) \leq n$, and 
(c) $F_{n}(X) \simeq(\Omega(X))^{*(n+1)}$, the $(n+1)$-fold join of $\Omega X$ with itself.

Proof Assertion (a) follows immediately from the construction. For parts (b) and (c), see [6]; these results also appear, from a different point of view, in [16].

\section{An Auxilliary Space}

Let $\widetilde{G}_{n}$ denote the homotopy pushout in the square

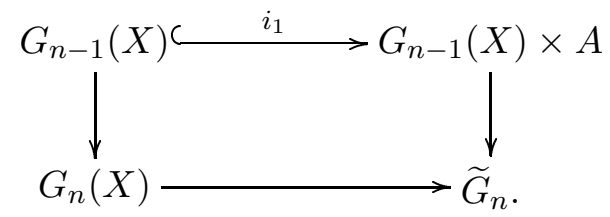

The maps $p_{n}: G_{n}(X) \rightarrow X$ and $1_{A}: A \rightarrow A$ piece together to give a map $\widetilde{p}_{n}: \widetilde{G}_{n} \rightarrow X \times A$. The space $\widetilde{G}_{n}$ and the map $\widetilde{p}_{n}$ play key roles in the forthcoming constructions; this section is devoted to establishing some of their properties.

\subsection{Category Properties of $\widetilde{G}_{n}$}

We begin by estimating the category of $\widetilde{G}_{n}$.

Proposition 5 For any noncontractible $A$ and $n>0$, $\operatorname{cat}\left(\widetilde{G}_{n}\right)<n+\operatorname{cat}(A)$.

Proof For simplicity in this proof, we write $F_{i}$ for $F_{i}(X)$ and $G_{i}$ for $G_{i}(X)$.

Let $\operatorname{cat}(A)=k$, so $A$ is a retract of another space $A^{\prime}$ with $\operatorname{cl}\left(A^{\prime}\right)=k$. Let $\widetilde{G}_{n}^{\prime}=G_{n} \cup G_{n-1} \times A^{\prime}$; clearly $\widetilde{G}_{n}$ is a homotopy retract of $\widetilde{G}_{n}^{\prime}$ and so it suffices to show that $\operatorname{cl}\left(\widetilde{G}_{n}^{\prime}\right)<n+k$. Let

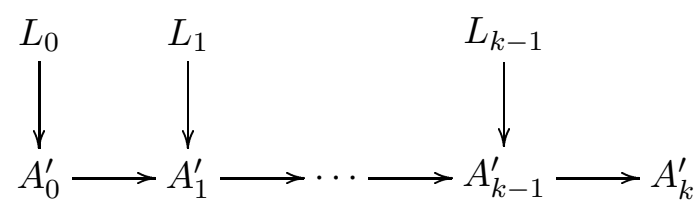

be a cone decomposition of $A^{\prime}$. We will also use the cone decomposition of $G_{n}$ given by the cofiber sequences $F_{i-1} \rightarrow G_{i-1} \rightarrow G_{i}$. According to a result of Baues 2] (see also [13, Prop. 2.9]), for each $i$ and $j$ there is a cofiber sequence

$$
F_{i-1} * L_{j-1} \longrightarrow G_{i} \times A_{j-1}^{\prime} \cup G_{i-1} \times A_{j}^{\prime} \longrightarrow G_{i} \times A_{j}^{\prime} .
$$


Now define subspaces $W_{s} \subseteq \widetilde{G}_{n}^{\prime}$ by the formula

$$
W_{s}= \begin{cases}\bigcup_{i+j=s} G_{i} \times A_{j}^{\prime} & \text { if } s \leq n \\ G_{n} \times A_{0}^{\prime} \cup\left(\bigcup_{\substack{i+j=s \\ i<n}} G_{i} \times A_{j}^{\prime}\right) & \text { if } s>n\end{cases}
$$

with the understanding that $A_{j}^{\prime}=A_{k}^{\prime}$ for all $j \geq k$. The cofiber sequences guaranteed by Baues' theorem can be pieced together with the given cone decompositions of $A^{\prime}$ and $G_{n}$ to give the cofiber sequences

$$
F_{s} \vee L_{s} \vee\left(\bigvee_{\substack{i+j=s-1 \\ i<n-1}} F_{i} * L_{j}\right) \longrightarrow W_{s} \longrightarrow W_{s+1}
$$

for each $s<\min \{n, k\}$; when $s \geq n$ we alter the cobase of the cofiber sequence by removing the $F_{s}$ summand, and when $s \geq k$ we must remove the summand $L_{s}$. Since $\widetilde{G}_{n}^{\prime}=W_{n+k-1}$, we have the result.

Next, we show that the map $\widetilde{p}_{n}: \widetilde{G}_{n} \rightarrow X \times A$ has one of the category-detecting properties of $p_{n}: G_{n}(X \times A) \rightarrow X \times A$.

Proposition 6 If $\operatorname{cat}(X \times A)=\operatorname{cat}(X)=n$, then $\widetilde{p}_{n}$ has a homotopy section.

Proof We follow [4] (see also [8, Thm. 2.7]) and define

$$
\widehat{G}_{n}^{\prime}(X \times A)=\bigcup_{i+j=n} G_{i}(X) \times G_{j}(A) .
$$

There is a natural map $h: \widehat{G}_{n}^{\prime}(X \times A) \rightarrow X \times A$ induced by the Ganea fibrations over $X$ and $A$. According to [4, Thm.2.3], $\operatorname{cat}(X \times A)=n$ if and only if $h$ has a homotopy section.

Each map $G_{i}(X) \times G_{j}(A) \rightarrow X \times A$ (with $j>0$ ) factors through $G_{i}(X) \times A$ and these factorizations are compatible because $p_{i+1}$ extends $p_{i}$. So $h$ factors as $\widehat{G}_{n}^{\prime}(X \times A) \rightarrow \widetilde{G}_{n} \rightarrow X \times A$. Therefore, if cat $(X \times A)=n$, then $h$, and hence $\widetilde{p}_{n}$, has a section.

\subsection{Comparison of $\widetilde{G}_{n}$ with $G_{n}(X) \times A$}

Let $j: \widetilde{G}_{n} \rightarrow G_{n}(X) \times A$ denote the natural inclusion map.

Proposition 7 Assume that $X$ is $(c-1)$-connected and that $A$ is $(r-1)$ connected. Then the homotopy fiber $F$ of the map $j$ is $(n c+r-2)$-connected. 
Proof There is a cofiber sequence

$$
\widetilde{G}_{n} \stackrel{j}{\longrightarrow} G_{n}(X) \times A \longrightarrow \Sigma F_{n-1}(X) \wedge A .
$$

Therefore the homotopy fiber of $j$ has the same connectivity as the space $\Omega\left(\Sigma F_{n-1}(X) \wedge A\right) \simeq \Omega\left(\Omega(X)^{* n} * A\right)$, namely $n c+r-2$.

Corollary 8 Assume $\operatorname{dim}(Z)<n c+r-2$ and let $f, g: Z \rightarrow \widetilde{G}_{n}$. Then $f \simeq g$ if and only if $j f \simeq j g$.

The proof is standard, and we omit it.

\subsection{New Sections from Old Ones}

Suppose that $\operatorname{cat}(X)=\operatorname{cat}(X \times A)=n$. By Proposition [6 there is a section $\sigma: X \times A \rightarrow \widetilde{G}_{n}$ of the map $\widetilde{p}_{n}: \widetilde{G}_{n} \rightarrow X \times A$. Define a new map $\sigma^{\prime}: X \rightarrow$ $G_{n}(X)$ by the diagram

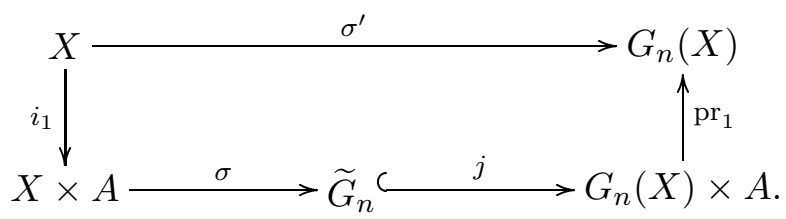

We need the following basic properties of $\sigma^{\prime}$.

Proposition 9 If $\operatorname{cat}(X \times A)=\operatorname{cat}(X)=n$, then

(a) $\sigma^{\prime}$ is a homotopy section of the projection $p_{n}: G_{n}(X) \rightarrow X$, and

(b) if $X$ is $(c-1)$-connected and $A$ is $(r-1)$-connected with $r>\operatorname{dim}(X)-$ $n c+2$, then the diagram

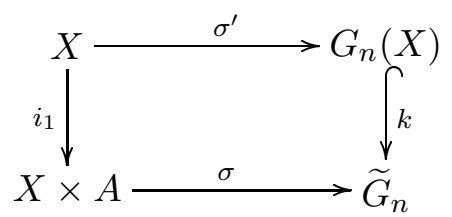

commutes up to homotopy. 
Proof First consider the diagram

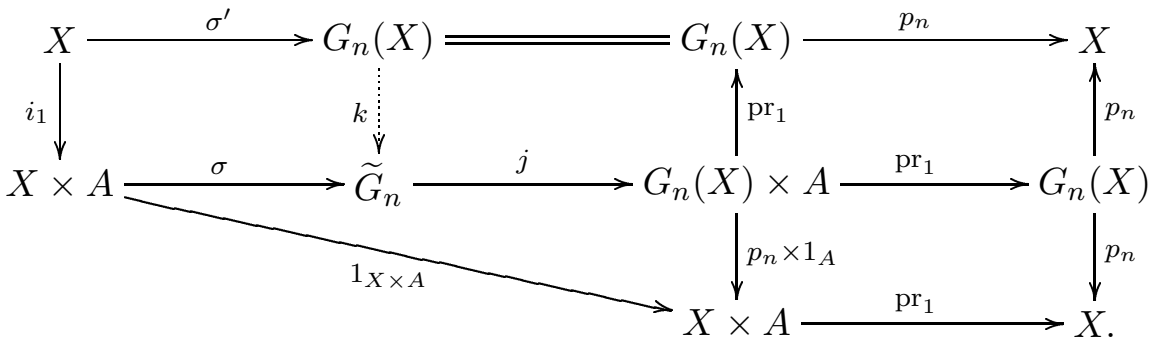

The diagram of solid arrows is evidently commutative. Therefore, we have $p_{n} \circ \sigma^{\prime} \simeq \operatorname{pr}_{1} \circ 1_{X \times A} \circ i_{1} \simeq 1_{X}$, proving (a).

To prove (b) we have to show that two maps $X \rightarrow \widetilde{G}_{n}$ are homotopic. Since $\operatorname{dim}(X)<n c+r-2$, it suffices by Corollary 8 to show that $j \circ\left(\sigma \circ i_{1}\right) \simeq$ $j \circ\left(k \circ \sigma^{\prime}\right)$. Since $\operatorname{pr}_{2} \circ j \circ\left(\sigma \circ i_{1}\right) \simeq * \simeq \operatorname{pr}_{2} \circ j \circ\left(k \circ \sigma^{\prime}\right)$, it remains to show that $\operatorname{pr}_{1} \circ j \circ\left(\sigma \circ i_{1}\right) \simeq \operatorname{pr}_{1} \circ j \circ\left(k \circ \sigma^{\prime}\right)$. But both of these maps are homotopic to $\sigma^{\prime}$.

\section{Proof of the Main Theorem}

Proof of Theorem [2 We have $n=\operatorname{cat}(X)=\operatorname{cat}(X \times A)$ by hypothesis. It follows from Proposition [6] that there is a section $\sigma: X \times A \rightarrow \widetilde{G}_{n}$ of the map $\widetilde{p}_{n}: \widetilde{G}_{n} \rightarrow X \times A$. We then get the section $\sigma^{\prime}: X \rightarrow G_{n}(X)$ that was constructed and studied in Section 2.3.

Consider the following diagram and the induced sequence of maps on the homotopy pushouts of the rows

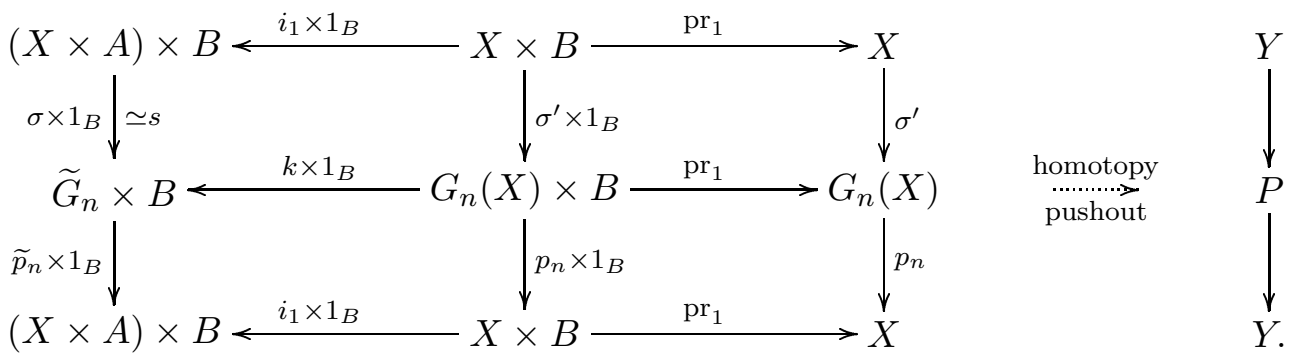

Proposition 9 implies that the upper left square commutes up to homotopy. Since $i_{1} \times 1_{B}$ is a cofibration, we can apply homotopy extension and replace the map $\sigma \times 1_{B}:(X \times A) \times B \rightarrow \widetilde{G}_{n} \times B$ with a homotopic map $s$ which makes 
that square strictly commute. All other squares are strictly commutative as they stand.

Since the composites $\left(\widetilde{p}_{n} \times 1_{B}\right) \circ\left(\sigma^{\prime} \times 1_{B}\right)$ and $p_{n} \circ \sigma^{\prime}$ are the identity maps and $\left(\widetilde{p}_{n} \times 1_{B}\right) \circ s$ is a homotopy equivalence, each vertical composite in the modified diagram is a homotopy equivalence. Thus $Y$ is a homotopy retract of $P$, and consequently $\operatorname{cat}(Y) \leq \operatorname{cat}(P)$.

The space $Y$ is the homotopy pushout of the top row in the diagram, which is the product of the homotopy pushout diagram

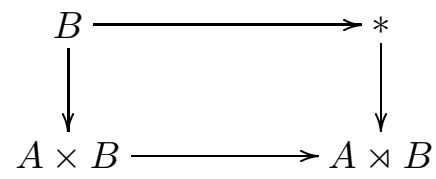

with the space $X$. Therefore $Y \simeq X \times(A \rtimes B)$ by Proposition 3. Since $Y$ is a homotopy retract of $P$, it follows that

$$
\operatorname{cat}(X \times(A \rtimes B)) \leq \operatorname{cat}(P),
$$

the proof will be complete once we establish that $\operatorname{cat}(P)<\operatorname{cat}(X)+\operatorname{cat}(A \rtimes B)$. This is accomplished in Lemma [10, which is proved below.

Lemma 10 The space $P$ constructed in the proof of Theorem 2 satisfies $\operatorname{cat}(P) \leq \operatorname{cl}(P)<\operatorname{cat}(X)+\operatorname{cat}(A \rtimes B)$.

Proof The space $\widetilde{G}_{n}$ is defined by the homotopy pushout square

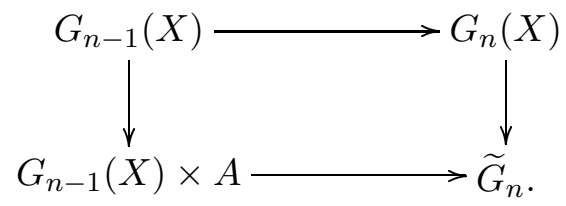

Take the product of this square with the space $B$ and adjoin the homotopy pushout square that defines $P$ to obtain the diagram

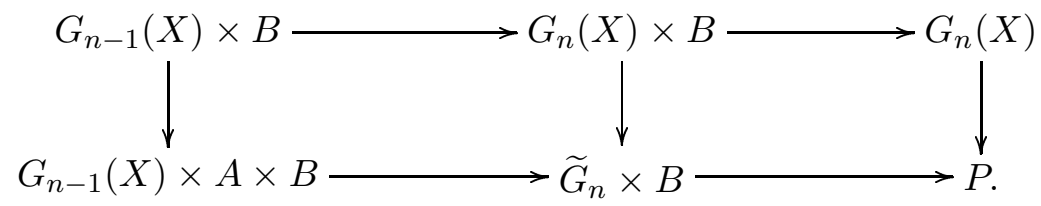

By [11, Lem. 13], the outer square

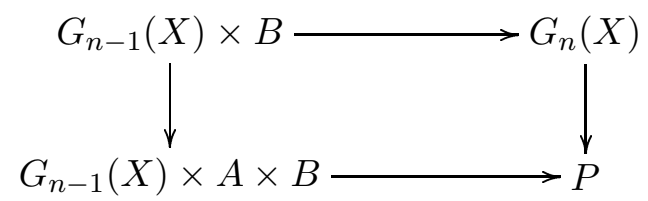


is also a homotopy pushout square. The top map is the composite

$$
G_{n-1}(X) \times B \stackrel{\mathrm{pr}_{1}}{\longrightarrow} G_{n-1}(X) \stackrel{\longrightarrow}{\longrightarrow} G_{n}(X),
$$

and so we have a new factorization into homotopy pushout squares:

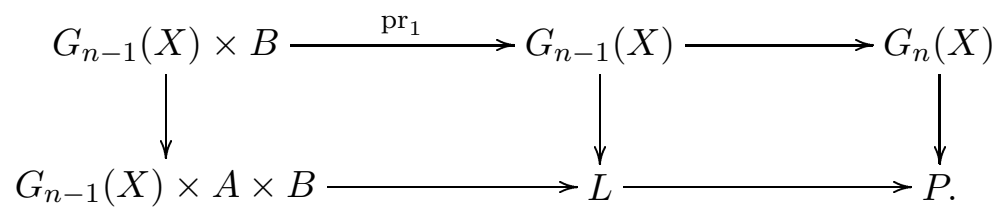

To identify the space $L$, observe that the left square is simply the product of the space $G_{n-1}(X)$ with the homotopy pushout square

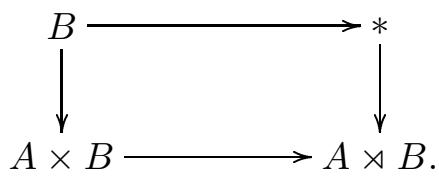

By Proposition 3, $L \simeq G_{n-1}(X) \times(A \rtimes B)$. Hence the right-hand square is the homotopy pushout square

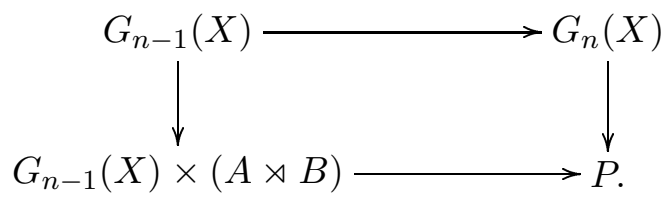

Therefore $\operatorname{cl}(P) \leq \operatorname{cat}(X)+\operatorname{cat}(A \rtimes B)$ by Proposition 5 .

\section{References}

[1] A. Bassi, Su alcuni nuovi invarianti della varietá topoligische, Annali Mat. Pura Appl. 16 (1935), 275-297. MathReview

[2] H. Baues, Iterierte Join-Konstruktion, Math. Zeit. 131 (1973), 77-84. MathReview

[3] I. Berstein and T. Ganea, The category of a map and a cohomology class, Fund. Math. 50 (1961/1962), 265-279. MathReview

[4] O. Cornea, G. Lupton, J. Oprea and D. Tanré, Lusternik-Schnirelmann category, Mathematical Surveys and Monographs 103, Amer. Math. Soc. Providence, RI (2003). MathReview

[5] T. Ganea, Some problems on numerical homotopy invariants, Symposium on Algebraic Topology (Battelle Seattle Res. Center, Seattle Wash., 1971), 23-30. Lecture Notes in Math., 249, Springer, Berlin, (1971). MathReview 
[6] T. Ganea, A generalization of the homology and homotopy suspension, Comm. Math. Helv. 39 (1965) 295-322. MathReview

[7] T. Ganea, Lusternik-Schnirelmann category and strong category, Ill. J. Math. 11 (1967) 417-427. MathReview

[8] N. Iwase, Ganea's conjecture on Lusternik-Schnirelmann category, Bull. London Math. Soc. 30 (1998), 623-634. MathReview

[9] N. Iwase $A_{\infty}$ method in Lusternik-Schnirelmann category, Topology 41 (2002) 695-723. MathReview

[10] I. M. James, On category, in the sense of Lusternik and Schnirelmann, Topology 17 (1977), 331-348. MathReview

[11] M. Mather, Pull-backs in homotopy theory, Canad. J. Math. 28 (1976), 225-263. MathReview

[12] D. Stanley, Spaces of Lusternik-Schnirelmann category $n$ and cone length $n+1$, Topology 39 (2000), 985-1019. MathReview

[13] D. Stanley, On the Lusternik-Schnirelmann category of maps, Canad. J. Math 54 (2002), 608-633. MathReview

[14] H. Scheerer, D. Stanley and D. Tanré, Fiberwise construction applied to Lusternik-Schnirelmann category, Israel J. Math. 131 (2002), 333-359. MathReview

[15] N. Steenrod, A convenient category of topological spaces, Mich. Math. J. 14 (1967), 133-152. MathReview

[16] A. Švarc, The genus of a fibered space, Translations of the AMS, 55 (1966), 49 - 140. MathReview

[17] L. Vandembroucq, Fibrewise suspension and Lusternik-Schnirelmann category, Topology 41 (2002), 1239-1258. MathReview

Faculty of Mathematics, Kyushu University, Ropponmatsu 4-2-1

Fukuoka 810-8560, Japan

Department of Mathematics and Statistics, University of Regina, College West 307.14

Regina, Saskatchewan, Canada

Department of Mathematics, Western Michigan University, 1903 W. Michigan Ave Kalamazoo, MI 49008, USA

Email: iwase@math.kyushu-u.ac.jp, stanley@math.uregina.ca, jeffrey.strom@wmich.edu

Received: 6 March 2004 